\title{
Avaliação da substituição do milho pelo resíduo seco da extração da fécula de mandioca sobre o desempenho de novilhas mestiças em confinamento
}

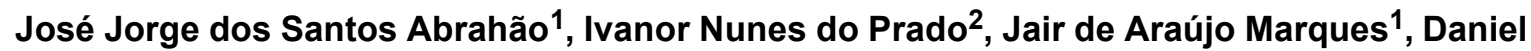 \\ Perotto $^{1}$, Simony Marta Bernardo Lugão ${ }^{1}$
}

\author{
${ }^{1}$ Instituto Agronômico do Paraná. \\ 2 Departamento de Zootecnia da Universidade Estadual de Maringá.
}

RESUMO - O objetivo neste trabalho foi avaliar o efeito da substituição total do milho pelo resíduo seco de mandioca sobre o desempenho de novilhas mestiças terminadas em confinamento. Foram utilizadas 30 novilhas mestiças com idade aproximada de 21 meses e peso vivo médio de $325 \mathrm{~kg}$, agrupadas em dois lotes experimentais e alojadas em duas baias. Os dois lotes foram submetidos a um período pré-experimental de 49 dias, no qual receberam silagem de sorgo, à vontade, e concentrado à base de farelo de soja, milho e uréia $(19,67 \%$ de proteína), na proporção de 1,05\% do peso corporal. Nesta fase, apresentaram ganho médio diário (GMD) de 1,08 kg semelhante para os lotes. Em seguida, iniciou-se o período experimental (57 dias), com os animais pesando, em média, $378 \mathrm{~kg}$. Um lote foi mantido sob a mesma dieta, enquanto o outro passou a receber o concentrado experimental, no qual o milho foi substituído pelo resíduo (18,12\% de proteína), na proporção de $1,18 \%$ do peso corporal, e silagem à vontade. O GMD dos animais diferiu entre as dietas, com 0,97 kg para os animais da dieta controle e $0,78 \mathrm{~kg}$ para aqueles que consumiram o concentrado com o resíduo seco. O desempenho, a ingestão voluntária de matéria seca e a conversão alimentar foram inferiores para os animais que consumiram o concentrado com resíduo seco. A substituição do milho pelo resíduo seco de mandioca reduziu o desempenho, a ingestão e a conversão alimentar.

Palavras-chave: alimentação, bovino de corte, subproduto da mandioca

\section{Effects of replacing corn with dry cassava by-product on production of feedlot crossbred heifers}

\begin{abstract}
The objective of this trial was to evaluate the total replacement of corn with a dry residue extracted from cassava starch on production of crossbred heifers in feedlot. Thirty crossbred heifers averaging 21 months of age and $325 \mathrm{~kg}$ of body weight were distributed in two experimental groups and then housed in two stalls. Both groups were submitted to a pretrial period of 49 days, in which animals received ad libitum sorghum silage and $1.05 \%$ of body weight as concentrate $(19.67 \%$ $\mathrm{CP}$ ) composed of soybean meal, corn, and urea. In this period, daily weigh gain (DWG) averaged $1.08 \mathrm{~kg}$ for both groups. The experimental period lasted 57 days with animals averaging $378 \mathrm{~kg}$ of body weight. At the beginning of the experimental period, one group remained receiving the pre-trial diet while the other was fed ad libitum sorghum silage and $1.18 \%$ of body weight as concentrate $(18.12 \% \mathrm{CP})$ containing dry cassava byproduct rather than corn. Daily weight gain was significantly higher for heifers fed corn-based concentrate $(0.97 \mathrm{~kg})$ compared to those receiving dry cassava byproduct-based concentrate $(0.78 \mathrm{~kg})$. In addition, dry matter intake and feed conversion were both lower on heifers fed the diet containing dry cassava byproduct.
\end{abstract}

Key Words: beef cattle, cassava by-product, feeding

\section{Introdução}

O confinamento de bovinos é uma prática importante nos sistemas de produção de gado de corte, pois permite maior flexibilidade no manejo dos animais, por meio do fornecimento de alimentação adequada nos períodos em que a disponibilidade de forragem é reduzida, possibilitando a adequada lotação das pastagens em relação à sua produção. Dessa forma, a utilização dos recursos alimentares é maximizada, aumentando a rentabilidade e liquidez da propriedade. No entanto, os custos com alimentação afetam os resultados econômicos dessa prática, tornando necessária a busca por alternativas alimentares mais baratas. A mandioca e os resíduos de seu processamento podem ser fontes alternativas de energia, uma vez que os grãos mais nobres e de maior custo são usados na alimentação humana e de animais não-ruminantes, que apresentam melhor aproveitamento desse tipo de alimento. 
A utilização da raspa de mandioca foi testada por Ferreira et al. (1989), que registraram ganhos de 1,1 a 1,2 kg por animal, ao utilizarem somente raspa de mandioca ou $50 \%$ de raspa $+50 \%$ de milho como principais fontes energéticas. Lorenzoni \& Mella (1994) avaliaram a inclusão do resíduo da produção de farinha, denominado "casquinha", em substituição ao milho desintegrado com palha e sabugo em rações para bovinos confinados e concluíram que esse resíduo poderia ser usado em até $45 \%$ do total da dieta.

A inclusão do resíduo de fecularia ensilado em dietas com silagem de sorgo, cana-de-açúcar ou ambos foi avaliada por Abrahão et al. (1997), que observaram ganho de peso superior a $1,00 \mathrm{~kg} / \mathrm{animal} / \mathrm{dia}$, em novilhas cruzadas consumindo elevada proporção de resíduo ensilado ( $45 \%$ da dieta constituída por resíduos + suplemento protéico).

A utilização da casca de mandioca em substituição ao milho em dietas para novilhas foi avaliada por Prado et al. (2000), que não encontraram diferença no ganho médio diário das novilhas alimentadas com a dieta com casca $(0,82 \mathrm{~kg})$, mas registraram ganho de $0,76 \mathrm{~kg}$ naquelas que consumiram a dieta com milho. Melhores resultados foram obtidos por Marques et al. (2000), que não notaram diferença na utilização do milho, da casca da mandioca, da farinha de varredura e da raspa de mandioca em dietas para novilhas cruzadas, observando ganhos diários de 1,60 kg por animal. Os autores atribuíram o elevado ganho a um possível efeito compensatório, uma vez que as novilhas, além de possuírem alto potencial genético para ganho de peso, foram mantidas a pasto e apresentavam ganhos de $0,40 \mathrm{~kg}$ por dia.

Todavia, os dados referentes ao uso de mandioca e de seus resíduos em substituição parcial ou total aos alimentos tradicionalmente utilizados na alimentação de bovinos em confinamento ainda são escassos e pouco conclusivos. Entre os inúmeros subprodutos originados do processamento da mandioca, existe o resíduo de fecularia, também denominado massa de fecularia ou massa de mandioca, resultante da extração do amido da mandioca por via úmida, com peso igual ou maior que o de raízes processadas pela indústria (Cereda, 1994). No processo de produção da fécula, após a extração do amido, obtém-se um resíduo com alta umidade $(85 \%)$, rico em fibras e amido residual, que, após secagem, apresenta a seguinte composição média: $9,52 \%$ de umidade, $0,66 \%$ de cinzas, $63,85 \%$ de amido, $0,83 \%$ de lipídios, $0,32 \%$ de nitrogênio e $14,88 \%$ de fibra bruta. Esse subproduto, pelos teores baixos de proteína e elevados de amido residual (não é removido durante o processo industrial), caracteriza-se como um alimento energético. Segundo Melotti(1972), o resíduo da industrialização da mandioca, resultante da extração da fécula, também denominado farelo de bagaço, apresenta $74,83 \%$ de NDT.
Esse resíduo apresenta elevado teor de umidade e, portanto, é bastante perecível, de difícil conservação e de transporte oneroso, o que limita sua utilização a locais próximos às indústrias. $\mathrm{O}$ resíduo é um material rico em carboidratos, de fácil e rápida fermentação ruminal (Caldas Neto et al., 2000) e efetivo como fonte energética para bovinos em confinamento (Abrahão, 1994). A secagem desse subproduto permite adequada conservação e transporte, otimizando sua utilização. O resíduo seco de fecularia, apesar de mais oneroso que o úmido, em razão dos custos envolvidos com prensagem e secagem, pode ser facilmente utilizado em misturas concentradas, reduzindo os custos com o manuseio do alimento nos sistemas de produção de animais em confinamento.

Este estudo foi realizado com o objetivo de avaliar o efeito da substituição total do milho pelo resíduo seco de fecularia sobre o consumo, a conversão alimentar e o desempenho de novilhas mestiças terminadas em confinamento.

\section{Material e Métodos}

O experimento foi realizado na Estação Experimental de Paranavaí, pertencente ao Instituto Agronômico do Paraná (IAPAR), localizada na região noroeste do Paraná.

Foram utilizadas 30 novilhas cruzas, com aproximadamente 21 meses de idade e peso médio de $325 \mathrm{~kg}$, provenientes do Programa de Cruzamentos do IAPAR e pertencentes aos seguintes grupos genéticos: 1/2Limousin + $1 / 4$ Simental $+1 / 4$ Nelore; $1 / 2$ Limuosin $+1 / 4$ Red Angus + $1 / 4$ Nelore; $1 / 2$ Marchigiana + $1 / 4$ Simental + 1/4Nelore; $3 / 4$ Marchigiana $+1 / 4$ Nelore; $1 / 2$ Limousin $+1 / 2$ Nelore e $1 / 2$ Marchigiana $+1 / 4$ Red Angus $+1 / 4$ Nelore. As novilhas foram agrupadas em dois lotes de 15 animais, de forma que ambos os lotes tivessem o mesmo número de animais $1 / 2 \mathrm{e}$ $3 / 4$ de sangue Europeu. As novilhas foram vacinadas contra febre aftosa e clostridioses, tratadas contra ectoparasitos, vermifugadas e identificadas com brincos plásticos na orelha esquerda. Em seguida, foram alojadas em duas baias coletivas de terra batida, cada uma com $24 \mathrm{~m}$ de largura por $67 \mathrm{~m}$ de comprimento, totalizando $1.608 \mathrm{~m}^{2}, \operatorname{com} 22,5 \mathrm{~m}$ lineares de cocho para alimento sólido e bebedouro com capacidade para $1.000 \mathrm{~L}$. Após o sorteio nos tratamentos e o alojamento nas respectivas baias, os animais foram submetidos a um período de adaptação às instalações e à alimentação, no qual foram fornecidos silagem de sorgo (à vontade, por três dias) e, posteriormente, concentrado (testemunha, à base de milho e soja), na proporção de 1,00 kg/dia, durante três dias. Em seguida, esta quantidade foi ajustada conforme o peso corporal $(1,05 \%$ do PV). 
Os tratamentos consistiram de dietas experimentais compostas por silagem de sorgo forrageiro (AG $2002^{\circledR}$ ), como volumoso, e pelos concentrados, à base de milho em grão (testemunha) ou resíduo seco da extração de fécula de mandioca (experimental), ambos com farelo de soja, uréia, vitaminas, minerais e aditivos. As dietas, formuladas segundo o AFRC (1993) para conter $12 \%$ de proteína e permitir ganho de $1,00 \mathrm{~kg} /$ animal $/ \mathrm{dia}$, foram enriquecidas com monensina sódica ( $30 \mathrm{mg} / \mathrm{kg}$ de MS da dieta). A composição média dos alimentos utilizados na formulação das dietas encontra-se na Tabela 1.

O experimento foi dividido em dois períodos: no primeiro (pré-experimental - 49 dias), os animais dos dois lotes foram alimentados com a mesma dieta, constituída de silagem de sorgo + concentrado testemunha, à base de farelo de soja e milho.

No segundo período (experimental-57 dias), um lote de animais recebeu o concentrado formulado com resíduo seco de fecularia em substituição ao milho (1,18 \% do PV) e o concentrado testemunha, com milho e soja (a 1,05\% do PV), conforme descrito na Tabela 2. Maior quantidade de concentrado com resíduo seco de fecularia foi fornecida para compensar seu baixo valor energético, ocasionado pela substituição do milho pelo resíduo.

Os animais foram alimentados diariamente pela manhã (9h), com metade do concentrado mais silagem de sorgo misturada ao concentrado, de modo a reduzir a seletividade pelos animais, è tarde (16h), com a outra metade, juntamente com a silagem. A silagem e os concentrados oferecidos diariamente foram pesados e os valores anotados, para o controle da oferta de silagem. Sempre que as sobras excederam $5 \%$ do total oferecido, e quando deterioradas, foram pesadas, anotadas e eliminadas. Amostras dos concentrados, da silagem, do resíduo de mandioca e das sobras foram coletadas semanalmente para análises dos terores de matéria seca (MS), matéria orgânica (MO), proteína bruta (PB), fibra bruta (FB), extrato etéreo (EE), extrativo não-nitrogenado $(\mathrm{ENN})$, matéria mineral (MM), fibra em detergente neutro
(FDN) e fibra em detergente ácido (FDA), conforme metodologia descrita por Silva \& Queiroz (2002). Os carboidratos totais (CHO) e não-fibrosos (CNF) foram determinados conforme descrição de Hall (2001).

Uma vez que o resíduo seco de mandioca, em comparação ao milho (Tabela 1), apresentou menores valores de PB e EE e maiores de FB, FDN, FDA e CHO, foi necessário o aumento da porcentagem de farelo de soja no concentrado com resíduo, para balancear a porcentagem de proteína. Para que a quantidade de energia oferecida pelo concentrado com resíduo fosse equivalente à do concentrado com milho, o percentual de concentrado fornecido em relação ao peso corporal foi um pouco maior. Dessa forma, os concentrados foram calculados considerando-se a composição da silagem, visandoà obtenção de dietas com aproximadamente $12 \%$ de PB (Tabela 3). Entretanto, foram encontradas diferenças entre os tratamentos, originadas da variação do consumo de MS do volumoso, que foi menor na dieta com o resíduo (Tabela 5), o que alterou sua relação volumoso:concentrado em relação à dieta com milho, na qual o volumoso participou com 55\% do total da MS. As diferenças nas proporções de volumoso e concentrado refletiram-se nas dietas consumidas, de modo que a dieta com resíduo seco apresentou maiores valores de PB, FB, FDN e FDA, enquanto aquela com milho, maiores valores de EE, ENN e CNF.

As quantidades de concentrados foram ajustadas após a pesagem dos animais, calculando-se as quantidades para cada lote conforme o percentual estabelecido anteriormente. Disponibilizou-se aos animais mistura mineral, à vontade, composta por cloreto de sódio e microelementos, apresentando a seguinte composição (por kg do produto): $\mathrm{Zn}(5000 \mathrm{mg}$ ), $\mathrm{Cu}$ (2000 mg), Co (30 mg), Se (67mg), I (155 mg)e Na (387 g).

Os animais foram pesados sempre pela manhã, após jejum de sólidos de 16 horas, ao início, aos 28 dias e aos 49 dias do período pré-experimental (início do período experimental) e aos 28 e 57 dias do período experimental.

$\mathrm{O}$ delineamento experimental foi o inteiramente casualisado, com dois tratamentos e quinze repetições. Os

Tabela 1 - Teores médios de PB, EE, MM, ENN, FDN, FDA, CNF e CHO dos alimentos concentrados e da silagem de sorgo (\% na MS) Table 1 - Average contents of CP, EE, MM, NFE, NDF, ADF, NFC, COH of concentrates components and shorgum silage (DM\%)

\begin{tabular}{|c|c|c|c|c|c|c|c|c|}
\hline $\begin{array}{l}\text { Componente } \\
\text { Item }\end{array}$ & $\begin{array}{r}\mathrm{PB} \\
C P\end{array}$ & $\begin{array}{l}\mathrm{EE} \\
E E\end{array}$ & $\begin{array}{l}\mathrm{MM} \\
\text { Ash }\end{array}$ & $\begin{array}{l}\text { ENN } \\
N F E\end{array}$ & $\begin{array}{l}\mathrm{FDN} \\
N D F\end{array}$ & $\begin{array}{l}\mathrm{FDA} \\
A D F\end{array}$ & $\begin{array}{l}\mathrm{CNF} \\
N F C\end{array}$ & $\begin{array}{l}\mathrm{CHO} \\
\mathrm{COH}\end{array}$ \\
\hline $\begin{array}{l}\text { Resíduo seco de mandioca } \\
\text { Cassava by-product }\end{array}$ & 1,59 & 0,19 & 1,12 & 80,65 & 28,83 & 22,96 & 68,27 & 97,10 \\
\hline $\begin{array}{l}\text { Milho grão } \\
\text { Corn grain }\end{array}$ & 9,13 & 3,67 & 1,11 & 84,18 & 12,08 & 3,01 & 74,01 & 86,09 \\
\hline $\begin{array}{l}\text { Farelo de soja } \\
\text { Soybean meal }\end{array}$ & 51,31 & 2,86 & 6,33 & 34,62 & 12,54 & 8,37 & 26,96 & 39,50 \\
\hline $\begin{array}{l}\text { Silagem de sorgo } \\
\text { Sorghum silage }\end{array}$ & 4,64 & 1,22 & 2,48 & 62,46 & 55,06 & 35,90 & 36,60 & 1,66 \\
\hline
\end{tabular}


Tabela 2 - Composição dos concentrados e teores de PB, EE, MM, ENN, FDN, FDA, CHO e CNF (\% na MS)

Table 2 - Concentrate composition and contents of CP, EE, ash, NFE, $N D F, A D F, C H O$ and NFC (DM\%)

\begin{tabular}{|c|c|c|c|}
\hline $\begin{array}{l}\text { Componente } \\
\text { Item }\end{array}$ & $\begin{array}{r}\mathrm{Co} \\
\mathrm{co} \\
\text { Corn }\end{array}$ & $\begin{array}{l}\text { ncentrado } \\
\text { m milho } \\
\text { concentrate }\end{array}$ & $\begin{array}{c}\text { Concentrado } \\
\text { com resíduo } \\
\text { Cassava concentrate }\end{array}$ \\
\hline $\begin{array}{l}\text { Resíduo seco de mandioca } \\
\text { Dry cassava by-product }\end{array}$ & & - & 73,77 \\
\hline $\begin{array}{l}\text { Milho grão } \\
\text { Corn grain }\end{array}$ & & 82,54 & - \\
\hline $\begin{array}{l}\text { Farelo de soja } \\
\text { Soybean meal }\end{array}$ & & 13,85 & 23,22 \\
\hline $\begin{array}{l}\text { Uréia } \\
\text { Urea }\end{array}$ & & 1,80 & 1,80 \\
\hline $\begin{array}{l}\text { Calcário calcítico } \\
\text { Limestone }\end{array}$ & & 1,40 & 0,50 \\
\hline $\begin{array}{l}\text { Fosfato bicálcico } \\
\text { Dicalcium phosphate }\end{array}$ & & 0,30 & 0,60 \\
\hline $\begin{array}{l}\text { Rumensin } \\
\text { Rumensin }^{1}\end{array}$ & & 0,06 & 0,06 \\
\hline $\begin{array}{l}\text { Premix vitamínico } \\
\text { Vitamin premix }\end{array}$ & & 0,05 & 0,05 \\
\hline
\end{tabular}

Composição química

Chemical composition

\begin{tabular}{lrr}
\hline PB (CP) & 19,67 & 18,12 \\
EE (EE) & 3,42 & 0,80 \\
MM (Ash) & 1,79 & 2,29 \\
ENN (NFE) & 74,27 & 67,53 \\
FDN (NDF) & 11,70 & 24,18 \\
FDA (ADF) & 3,64 & 18,88 \\
CHO (COH) & 76,53 & 80,80 \\
CNF (NFC) & 64,82 & 56,62 \\
\hline
\end{tabular}

${ }^{1}$ Rumensin ${ }^{\circledR}$ Laboratório Elanco: $100 \mathrm{mg}$ de monensina por grama do produto.

2 Rovimix ${ }^{\circledR}$ Laboratório Roche: vitamina A 20.000.000 UI, vitamina D3 2.000.000 UI, vitamina E $80.000 \mathrm{UI}$ por kg do produto.

1 Rumensin $\circledast$ Elanco Laboratories: $100 \mathrm{mg}$ of monensin pergram of product.

2 Rovimix $\circledast$ Roche Lab: vitamin A 20.000.000 IU, vitamin D3 2.000.000 IU, vitamin E 80.000 IU per kg of product.

Tabela 3 - Porcentagem de silagem e concentrado nas dietas e composição em PB, EE, MM, ENN, FDN, FDA, CHO e CNF (\% na MS)

Table 3 - Ingredient composition of diets and contents of $C P, E E$, ash, NFE, NDF, ADF, $\mathrm{COH}$, and NFC (\%DM)

\begin{tabular}{lcc}
\hline $\begin{array}{l}\text { Dieta } \\
\text { Diet }\end{array}$ & $\begin{array}{c}\text { Com milho } \\
\text { With corn }\end{array}$ & $\begin{array}{c}\text { Com resíduo seco } \\
\text { With cassava }\end{array}$ \\
\hline $\begin{array}{l}\text { Concentrado (\%) } \\
\text { Concentrate }\end{array}$ & 44,88 & 55,79 \\
$\begin{array}{l}\text { Silagem (\%) } \\
\text { Silage }\end{array}$ & 55,12 & 44,21
\end{tabular}

Composição química

Chemical composition

\begin{tabular}{lrr}
\hline PB (CP) & 11,38 & 12,16 \\
EE (EE) & 2,20 & 0,98 \\
MM (MM) & 2,17 & 2,37 \\
ENN (NFE) & 67,76 & 65,28 \\
FDN (NDF) & 35,60 & 37,83 \\
FDA (ADF) & 21,42 & 26,40 \\
CHO (CHO) & 84,25 & 84,49 \\
CNF (NFC) & 48,25 & 46,66 \\
\hline
\end{tabular}

dados de ganho de peso e dos pesos inicial e final foram submetidos à análise de variância e analisados pelo SAEG (UFV, 1997), utilizando-se o método dos quadrados mínimos, e as médias, comparadas pelo teste $\mathrm{F}$, a $5 \%$ de significância, conforme o modelo a seguir:

$$
\mathrm{Y}_{\mathrm{ij}}=\mu+\mathrm{t}_{\mathrm{i}}+\mathrm{e}_{\mathrm{ij}}
$$

em que $Y_{i j}=$ observação no animal j submetido ao tratamento $i$; $\mu=$ constante geral; $t_{i}=$ efeito do tratamento $i ; i=1 ; 2$; $\mathrm{e}_{\mathrm{ij}}=$ erro aleatório associado a cada observação $\mathrm{Y}_{\mathrm{ij}}$.

\section{Resultados e Discussão}

Os pesos médios dos animais dos dois lotes (Tabela 4), ao início do período pré-experimental, não diferiram entre si $(\mathrm{P}>0,05)$. Durante o período pré-experimental, as novilhas foram alimentadas com uma dieta única, à base de concentrado com milho (Tabela 2) e silagem de sorgo, e apresentaram desempenho semelhante (Tabela 4), com ganhos diários de 1,10 e $1,07 \mathrm{~kg}$, atendendo à expectativa previamente definida em função da dieta calculada (em torno de $1,00 \mathrm{~kg} / \mathrm{dia}$ ). Os ganhos desse período foram elevados, uma vez que os animais, ao início do período de confinamento, apresentavam bom estado corporal, compatível com o peso médio inicial, o que reduz a possibilidade de algum efeito de ganho compensatório.

O ganho de peso no período e o ganho médio diário durante o período experimental (Tabela 4 ) foram diferentes para as dietas avaliadas $(\mathrm{P}<0,05)$, com superioridade de $24 \%$ para a dieta com o concentrado testemunha à base de milho em relação àquela com o concentrado experimental à base de resíduo seco de fecularia.

Estes resultados corroboram os descritos por Ramos et al. (2000), que avaliaram o mesmo produto sob a forma úmida em diferentes níveis de substituição ao milho em dietas para novilhos machos inteiros e não encontraram diferença para ganhos, que variaram de $1,10 \mathrm{~kg} /$ dia para a dieta testemunha; 0,95 para a dieta com $33 \%$ de substituição; e 1,12 kg/dia para $66 \%$ de substituição. Contudo, quando a substituição do milho foi de $99 \%$, os ganhos foram de $0,75 \mathrm{~kg} / \mathrm{dia}$, inferiores aos das demais dietas.

Valores semelhantes foram reportados por Prado et al. (2000), que testaram o uso de casca de mandioca em substituição ao milho na dieta de novilhas em confinamento e não notaram diferença para os ganhos entre as dietas com casca $(0,82 \mathrm{~kg} / \mathrm{dia})$ ou com milho $(0,76 \mathrm{~kg} / \mathrm{dia})$. Os autores atribuíram o baixo ganho $(0,79 \mathrm{~kg} / \mathrm{dia})$ observado no experimento, independentemente das dietas, à reduzida ingestão voluntária, decorrente do baixo valor nutritivo da silagem utilizada e da ocorrência de estro durante o experimento. 
Tabela 4 - Pesos vivos inicial e final, ganho de peso no período (GPP), ganho médio diário (GMD) dos animais e coeficiente de variação (CV\%) nos períodos pré-experimental e experimental

Table 4 - Initial and final body weight, total gain in period (TGP), daily weight gain (DWG) and coefficient of variation (CV\%) in the pre-experimental and experimental periods

\begin{tabular}{|c|c|c|c|}
\hline \multirow{2}{*}{$\begin{array}{l}\text { Período pré-experimental } \\
\text { Pre-experimental period } \\
\text { Peso inicial, } \mathrm{kg} \\
\text { Initial weight, } \mathrm{kg}\end{array}$} & \multicolumn{2}{|c|}{$\begin{array}{c}\text { Dietas com milho } \\
\text { Corn diets }\end{array}$} & \multirow{2}{*}{$\begin{array}{l}\text { CV\% } \\
10,91\end{array}$} \\
\hline & 326,27 & 323,47 & \\
\hline $\begin{array}{l}\text { Peso final, } \mathrm{kg} \text { (49 dias) } \\
\text { Final weight, } \mathrm{kg} \text { (49 days) }\end{array}$ & 380,20 & 376,07 & 11,07 \\
\hline $\begin{array}{l}\mathrm{GPP}, \mathrm{kg} \\
T G P, \mathrm{~kg}\end{array}$ & 53,93 & 52,60 & 33,87 \\
\hline $\begin{array}{l}\mathrm{GMD}, \mathrm{kg} / \mathrm{dia} \\
D W G, \mathrm{~kg} / \text { day }\end{array}$ & 1,10 & 1,07 & 33,31 \\
\hline $\begin{array}{l}\text { Período experimental } \\
\text { Experimental period }\end{array}$ & $\begin{array}{l}\text { Dieta com milho } \\
\text { Diet with corn }\end{array}$ & $\begin{array}{c}\text { Dieta com resíduo } \\
\text { Diet with cassava }\end{array}$ & \\
\hline $\begin{array}{l}\text { Peso inicial, } \mathrm{kg} \\
\text { Initial weight, } \mathrm{kg}\end{array}$ & 380,20 & 376,07 & 11,07 \\
\hline $\begin{array}{l}\text { Peso final, } \mathrm{kg} \text { (57dias) } \\
\text { Final weight, } \mathrm{kg} \text { (57 days) }\end{array}$ & 433,54 & 418,60 & 11,46 \\
\hline $\begin{array}{l}\mathrm{GPP}, \mathrm{kg} \\
T G P, \mathrm{~kg}\end{array}$ & $53,33 \mathrm{a}$ & $42,53 b$ & 29,87 \\
\hline $\begin{array}{l}\mathrm{GMD}, \mathrm{kg} \\
D W G, \mathrm{~kg}\end{array}$ & $0,93 \mathrm{a}$ & $0,75 b$ & 29,26 \\
\hline
\end{tabular}

Médias na linha seguidas de letras distintas diferem $(P<0,05)$ pelo teste $F$. Means in a row, followed by different letters, differ $(P<0.05)$ by $F$ test.

Marques et al. (2000) compararam dietas com milho, casca da mandioca, farinha de varredura e raspa da mandioca na alimentação de novilhas cruzadas e não verificaram diferenças no desempenho, registrando ganhos médios diários de $1,60 \mathrm{~kg} /$ animal. Esses autores atribuíram o elevado desempenho ao potencial genético para ganho de peso dos animais mestiços e ao possível efeito compensatório, visto que, anteriormente ao experimento, as novilhas foram mantidas a pasto e apresentavam ganhos de $0,40 \mathrm{~kg} / \mathrm{dia}$.

Os menores ganhos das novilhas durante o período experimental, em relação ao período pré-experimental, podem ser atribuídos ao estado corporal ao longo do período préexperimental e ao elevado peso dos animais no início da avaliação das dietas - $380 \mathrm{~kg}$ para os animais testemunhas e $376 \mathrm{~kg}$ para os do tratamento experimental. Em animais confinados por longos períodos, as taxas de ganho tendem a decrescer (Esteves, 1997), provavelmente em razão do aumento do grau de acabamento, que eleva a quantidade de energia depositada nos tecidos (NRC, 1996), observando-se menores ganhos com mesma quantidade de alimento ingerido.

Tendência semelhante foi observada por Leme et al. (2000), que registraram redução no ganho médio diário à medida que aumentou o período de permanência dos animais no confinamento. Os valores foram de 1,18; 1,07 e $0,98 \mathrm{~kg}$ para os confinados por 67,117 e 151 dias, respectivamente. $\mathrm{O}$ aumento do peso corporal resulta em maiores necessidades de energia para mantença, que, quando não são compensadas pelo aumento na ingestão de alimento, provocam redução da disponibilidade de energia para ganho.

Os valores para consumo de MS e conversão da MS (Tabela 5) não foram submetidos à análise estatística, pois esses dados foram determinados para o lote de animais, sendo, portanto, valores médios sem repetições. Entretanto, o consumo de MS pelos animais alimentados com a dieta testemunha foi $10,40 \%$ maior que o daqueles que consumiram o resíduo seco de fecularia.

No experimento de Marques et al. (2000), os consumos de MS determinados para novilhas com peso médio inicial de $365 \mathrm{~kg}$ foram de $11,70 \mathrm{~kg}$ (dieta com milho), 10,10 kg (dieta com casca de mandioca), 9,50 kg (dieta com raspa de mandioca) e $8,50 \mathrm{~kg}$ (dieta com farinha de varredura), ou seja, houve decréscimo no consumo das dietas com resíduos de mandioca, que, no caso da farinha de varredura, foi atribuído pelos autores, à baixa porcentagem de extrato etéreo, à pulverulência do produto e à sua tendência em colar na língua e na boca dos animais, dificultando a ingestão. Contudo, quando o consumo foi expresso em porcentagem do peso corporal, determinaram-se valores que oscilaram de $2,70 \%$ do peso (dieta com milho) a $2,10 \%$ (dieta com farinha de varredura), superiores aos obtidos neste experimento.

Valores para consumo de MS de dietas com subprodutos de mandioca próximos aos deste ensaio foram determinados 
Tabela 5 - Ingestão de matéria seca (MS) e conversão alimentar da matéria seca no período experimental

Table 5 - Drymatter (DM) intake and feed conversion in the experimental period

\begin{tabular}{|c|c|c|}
\hline $\begin{array}{l}\text { Variável } \\
\text { Variable }\end{array}$ & $\begin{array}{c}\text { Dieta com } \\
\text { milho } \\
\text { Diet with } \\
\text { corn }\end{array}$ & $\begin{array}{c}\text { Dieta com } \\
\text { resíduo } \\
\text { Diet with } \\
\text { cassava }\end{array}$ \\
\hline $\begin{array}{l}\text { Ingestão de } \mathrm{MS}, \mathrm{kg} / \mathrm{dia} \\
\text { DM Intake, } \mathrm{kg} / \text { day }\end{array}$ & 8,34 & 7,55 \\
\hline $\begin{array}{l}\text { Ingestão de MS, por } 100 \mathrm{~kg} \text { de peso } \\
\text { DM Intake, } 100 \mathrm{~kg} \text { of body weight }\end{array}$ & 2,05 & 1,90 \\
\hline $\begin{array}{l}\text { Conversão alimentar, } \mathrm{kg} \mathrm{MS} / \mathrm{kg} \text { de peso } \\
\text { Feed conversion, } \mathrm{kg} \mathrm{DM} / \mathrm{kg} \text { of body weight }\end{array}$ & $8,9: 1$ & $10,12: 1$ \\
\hline
\end{tabular}

por Prado et al. (2000), que encontraram valores de ingestão de $2,15 \%$ para dieta com casca de mandioca e de $2,09 \%$ do PV para a dieta contendo milho e levedura como fonte de proteína.

A conversão alimentar da MS foi melhor nos animais que consumiram a dieta contendo milho que naqueles alimentados com a dieta com resíduo de fecularia, visto que o GMD dos animais alimentados com a dieta testemunha $(0,93 \mathrm{~kg} / \mathrm{dia})$ foi maior que o daqueles alimentados com a dieta experimental $(0,75 \mathrm{~kg} / \mathrm{dia})$, apesar do menor consumo de MS (em porcentagem do peso corporal) dos animais submetidos à dieta experimental. A conversão alimentar determinada nas dietas avaliadas pode ser considerada baixa e provavelmente resultou do peso dos animais ao início do período experimental (380,20 e 376,07/média dos tratamentos), o que provocou aumento das exigências de mantença, e do elevado grau de acabamento dessas novilhas, o que, no entanto, aumentaria a demanda de energia em relação à composição dos tecidos sintetizados.

Prado et al. (2000) avaliaram a conversão alimentar de uma dieta com silagem de milho e concentrado com milho em grão $(8,32: 1)$ e de outra com casca de mandioca $(7,43: 1)$ e demonstraram que a conversão obtida para a dieta contendo milho na fração concentrada foi semelhante à encontrada neste trabalho, enquanto a da dieta com casca de mandioca foi melhor que a da dieta com milho e à obtida neste estudo.

Melhor eficiência alimentar foi encontrada por Marques et al. (2000), que determinaram conversões de 5,9:1 para dieta com casca de mandioca, 6,3:1 para dieta com raspa de mandioca e 7,3:1 para a dieta com a farinha de varredura. Contudo, nesse trabalho, os elevados ganhos foram ocasionados pela curta duração do experimento e pelo fato de que, anteriormente, as novilhas estavam em pastagens que não permitiam elevados ganhos $(0,40 \mathrm{~kg} / \mathrm{dia})$.

\section{Conclusões}

A substituição do milho pelo resíduo seco da extração da fécula de mandioca na dieta provocou redução no ganho de peso e no consumo, resultando em pior conversão alimentar.

\section{Literatura Citada}

ABRAHÃO, J.J.S. Utilização do resíduo da extração da fécula da mandioca ensilado, associado torta de soja e uréia na alimentação de novilhas cruzadas. In: REUNIÃO ANUAL DA SOCIEDADE BRASILEIRA DE ZOOTECNIA, 31., 1994, Maringá. Anais... Maringá: Sociedade Brasileira de Zootecnia, 1994.

ABRAHÃO, J.J.S.; PEROTTO, D.; MOLETTA, J.L. Avaliação da substituição da silagem de sorgo por cana-de-açúcar em dietas com resíduos de fecularia no desempenho de novilhas. In: REUNIÃO ANUAL DA SOCIEDADE BRASILEIRA DE ZOOTECNIA, 34., 1997, Juiz de Fora. Anais... Juiz de Fora: Sociedade Brasileira de Zootecnia, 1997.

AGRICULTURAL AND FOOD RESEARCH COUNCIL - AFRC. Technical committee on responses to nutrients: energy and protein requirements of ruminants. Wallingford: CAB International, 1993. 159p.

CALDAS NETO, S.F.; ZEOULA, L.M.; BRANCO, A.F. et al. Mandioca e resíduos das farinheiras na alimentação de ruminantes: digestibilidades total e parcial. Revista Brasileira de Zootecnia, v.26, p.2099-2108, 2000.

CEREDA, M.P. Caracterização dos resíduos da industrialização da mandioca. In: CEREDA, M.P. Resíduos da industrialização da mandioca. Botucatu, 1994. p.11-50.

ESTEVES, S.N. Confinamento para produção de novilho precoce. In: Intensificação de bovinocultura de corte - estratégias de alimentação e terminação. São Carlos: Embrapa, 1997. p.44-57. (Documento, 27)

FERREIRA, J.J.; MARQUES NETO, J.; MIRANDA, C.S. Efeito do milho, sorgo e raspa de mandioca na ração, sobre o desempenho de novilhos confinados. Revista da Sociedade Brasileira de Zootecnia, v.18, n.4, p.306-313, 1989.

HALL, M.B. Recent advances in non-NDF carbohydrates for the nutrition of lactating cows. In: SIMPÓSIO INTERNACIONAL EM BOVINOCULTURA DE LEITE: NOVOS CONCEITOS EM NUTRIÇÃO, 2., 2001, Lavras. Anais... Lavras: Universidade Federal de Lavras, 2001. p.139-148.

LEME, P.R.; BOIN, C.; MARGARIDO, R.C.C. et al. Desempenho em confinamento e características de carcaça de bovinos machos de diferentes cruzamentos abatidos em três faixas de peso. Revista da Sociedade Brasileira de Zootecnia, v.29 n.6, p.2347-2353, 2000 (supl. 2).

LORENZONI, W.R.; MELLA, S.C. Avaliação do resíduo obtido da lavagem de raiz de mandioca como alimento energético para bovinos. In: CEREDA, M.P. (Ed.) Resíduos da industrialização da mandioca. Botucatu: 1994. p.91-100.

MARQUES, J.A.; PRADO, I.N.; ZEOULA, L.M. et al. Avaliação da mandioca e seus resíduos industriais em substituição ao milho no desempenho de novilhas confinadas. Revista Brasileira de Zootecnia, v.29, n.5, p.1528-1536, 2000.

MELOTTI, L. Contribuição para o estudo da composição química e valor nutritivo dos resíduos da industrialização da mandioca, Manhiot utilissima, Pohl., no estado de São Paulo. Boletim da Industria Animal, v.29, n.2, p.339-349, 1972.

NATIONAL RESEARCH COUNCIL - NRC. Nutrients requirements of beef cattle. 7.ed. Washington, D.C.: National Academy Press, 1996. 244p.

PRADO, I N.; MARTINS, A.S.; ALCALDE, C.R. et al. Desempenho de novilhas alimentadas com rações contendo milho ou casca de mandioca como fonte energética e farelo de algodão ou levedura 
como fonte protéica. Revista Brasileira de Zootecnia, v.29, n.1, p.278-287, 2000

RAMOS, P.R.; PRATES, E.R.; FONTANELLI, R.S. et al. Uso do bagaço de mandioca em substituição ao milho no concentrado para bovinos em crescimento. 2. Digestibilidade aparente, consumo de nutrientes digestíveis, ganho de peso e conversão alimentar. Revista Brasileira de Zootecnia, v.29, n.1, p.300$305,2000$.

SILVA, D.J.; QUEIROZ, A.C. Análise de alimentos: métodos químicos e biológicos. 3.ed. Viçosa, MG: Universidade Federal de Viçosa, 2002. 166p.
UNIVERSIDADE FEDERAL DE VIÇOSA - UFV. SAEG - Sistema de Análises Estatísticas e Genéticas. Versão 7.1. Viçosa, MG: 1997. 150p. (Manual do usuário).

Recebido: 01/07/04 Aprovado: 04/08/05 